\title{
Potent anti-tumour activity of a novel conditionally replicating adenovirus for melanoma via inhibition of migration and invasion
}

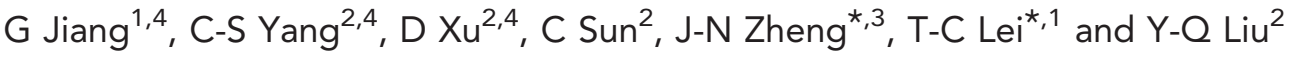 \\ ${ }^{1}$ Department of Dermatology, Renmin Hospital of Wuhan University, Wuhan 430060, Hubei Province, China; ${ }^{2}$ Department of \\ Dermatology, Affiliated Hospital of Xuzhou Medical College, Xuzhou 221002, Jiangsu Province, China and ${ }^{3} J i a n g s u$ Key \\ Laboratory of Biological Cancer Therapy, Xuzhou Medical College, Xuzhou 221002, Jiangsu Province, China
}

Background: Conditionally replicating adenoviruses (CRAds) represent a novel class of oncological therapeutic agents. One strategy to ensure tumour targeting is to place the essential viral genes under the control of tumour-specific promoters. Ki67 has been selected as a cancer gene therapy target, as it is expressed in most malignant cells but is barely detectable in most normal cells. This study aimed to investigate the effects of a Ki67 promoter-controlled CRAd (Ki67-ZD55-IL-24) on the proliferation and apoptosis of melanoma cells.

Methods: Melanoma cells were independently treated with Ki67-ZD55-IL-24, ZD55-IL-24, Ki67-ZD55, and ZD55-EGFP. The cytotoxic potential of each treatment was assessed using cell viability measurements. Cell migration and invasion were assayed using cell migration and invasion assays. Apoptosis was assayed using the annexin V-FITC assay, western blotting, reverse transcriptase PCR (RT-PCR), haematoxylin and eosin (H\&E) staining, and the TUNEL assay.

Results: Our results showed that Ki67-ZD55-IL-24 had significantly enhanced anti-tumour activity as it more effectively induced apoptosis in melanoma cells than the other agents. Ki67-ZD55-IL-24 also caused the most significant inhibition of cell migration and invasion of melanoma cells. Furthermore, apoptosis was induced more effectively in melanoma xenografts in nude mice.

Conclusions: This strategy holds promising potential for the further development of an effective approach to treat malignant melanoma.

Melanoma is among the most malignant types of tumours whose incidence has increased the fastest over the last few decades. The prognosis for melanoma patients with distant metastases is dismal, with a median survival time of 6-9 months (Sinnberg et al, 2009). Metastatic melanoma is generally treated with systemic chemotherapy, immunotherapy, and radiotherapy. The reported long-term survival rate of melanoma is not encouraging due to its chemoresistance and rapid metastasis. Given the limitations of the current therapies for metastatic melanoma, there is an urgent need to develop novel treatments.

$\mathrm{Ki} 67$ is a nuclear protein that is associated with cell proliferation (Zheng et al, 2006). Cells express Ki67 during the G1, S, G2, and $M$ phases but not during the resting phase, G0 (Beresford et al, 2006). The Ki67 labelling index is an independent predictor of disease progression and recurrence in carcinomas including melanoma, and it is used as a grade index and prognostic marker

\footnotetext{
*Correspondence: Professor T-C Lei; E-mail: tchlei@whu.edu.cn or Professor J-N Zheng; E-mail: dr.guanjiang@gmail.com

${ }^{4}$ These authors contributed equally to this work
}

Received 4 April 2013; revised 4 March 2014; accepted 6 March 2014; published online 8 April 2014

(c) 2014 Cancer Research UK. All rights reserved 0007-0920/14 
(Cheang et al, 2009; Yerushalmi et al, 2010; Liu et al, 2011; Vaisanen et al, 2011). Ki67 is highly overexpressed in a number of cancers including melanoma. As an independent prognostic factor, the expression level of Ki67 has been positively correlated with some clinicopathological variables in patients (Rioux-Leclercq et al, 2000). Thus, Ki67 has been selected as a target of cancer gene therapy because it is present in most malignant cells but scarcely detected in most normal cells and has been related to cell proliferation.

Melanoma differentiation associated gene-7/interleukin-24 (mda-7/IL-24) belongs to the IL-10 family of cytokines (Jiang et al, 1996). Interleukin-24 has been identified by using subtraction hybridisation of cDNA libraries from actively proliferating human HO-1 melanoma cells $v s$ interferon-band mezerein-treated HO- 1 cells (Eager et al, 2008). In addition, many studies have established IL-24 as a promising therapeutic candidate with potent anti-tumour, anti-angiogenic, and cytokine activities (Emdad et al, 2009).

Conditionally replicating adenoviruses (CRAds) represent a novel class of therapeutic agents in cancer treatment (Liu, 2006). As an oncolytic transgene delivery system, CRAds selectively replicate in and lyse tumour cells, amplify therapeutic gene expression, and function in the tumour microenvironment (Cody and Douglas, 2009). There are two major strategies to generate oncolytic adenoviruses: one strategy is to delete the viral element that is necessary for viral replication in normal cells but is dispensable in tumour cells, such as ONYX-015 or ZD55 with deletion of the E1B-55KDa gene (Bischoff et al, 1996; Liu, 2006). The other strategy is to drive the necessary gene for viral replication by a tumour-specific promoter (Yu et al, 2001). For example, the Ki67 promoter has been used in our laboratory to control E1A of adenovirus to construct oncolytic adenoviruses, which show tumour-selective replication and potent anti-tumour efficacy (Wang et al, 2011; Pei et al, 2012).

In this study, combining virtues of the two strategies above, IL-24 was inserted into Ki67-ZD55, a CRAd with a 55-kDa deletion in the E1B gene, which was driven by the Ki67 promoter and a BglII cloning site to insert foreign therapeutic genes to form Ki67-ZD55IL-24. We explored the anti-tumour effects of Ki67-ZD55-IL-24 both in vitro and in vivo. Our data suggest that Ki67-ZD55-IL-24 can be used as a potential therapeutic strategy for the treatment of melanoma.

\section{MATERIALS AND METHODS}

Cell cultures. The human melanoma A375 and M14 cell lines were purchased from the Shanghai Cell Collection (Shanghai, China). Normal human lung fibroblast (NHLF) cells were purchased from Shanghai Cell Collection. Cells were cultured in Dulbecco's modified Eagle's medium (DMEM; GIBCO BRL, Grand Island, NY, USA) supplemented with $10 \%$ heat-inactivated fetal bovine serum (FBS; GIBCO-BRL), $4 \mathrm{~mm}$ glutamine, $50 \mathrm{U} \mathrm{ml}^{-1}$ penicillin, and $50 \mu \mathrm{g} \mathrm{ml}^{-1}$ streptomycin at $37^{\circ} \mathrm{C}$ in a humidified atmosphere with $5 \% \mathrm{CO}_{2}$. The cells were screened routinely to verify lack of mycoplasma contamination in the log phase of growth.

Virus construction, production, and transfection. The recombinant adenoviruses used in this study have been previously described (Zhao et al, 2005; Jiang et al, 2013), which included Ki67 promoter-controlled ZD55 carrying IL-24 (Ki67-ZD55-IL-24), Ki67 promoter-controlled ZD55 (Ki67-ZD55), ZD55 carrying IL-24 (ZD55-IL-24), and ZD55 carrying enhanced green fluorescence protein (ZD55-EGFP). The Ki67 promoter used in this study was constructed previously (Wang et al, 2011; Pei et al, 2012). Viral plaques appeared 9-12 days after transient transfection.
These recombinant adenoviruses were verified by reverse transcriptase PCR (RT-PCR). Viruses were plaque purified and propagated on HEK293 cells, and functional plaque-forming unit (PFU) titres were determined by a plaque assay on HEK293 cells. The PFU titres of the adenoviruses were $3.5 \times 10^{10} \mathrm{PFU}$ per $\mathrm{ml}$.

Reverse transcriptase-PCR. Total cellular RNA was purified using a total RNA isolation system and RT-PCR using the Access RT-PCR system (Promega, Madison, WI, USA). The nucleotide sequences of the IL-24 primers were as follows: the upstream and downstream primers were 5'-TCTACCGGAAGAGTGTCTG GAGCAA- $3^{\prime}$ and 5'-GCTCCCACGACGTAGTCCATGTTCA-3', respectively. The nucleotide sequences of the GAPDH primers were as follows: the upstream and downstream primers were $5^{\prime}$-ATGGCATGCGACTTGAGT- $3^{\prime}$ and $5^{\prime}$-AGCGGTACCGCTCC TTAG- $3^{\prime}$, respectively. The thermal cycles were $96^{\circ} \mathrm{C}$ for $5 \mathrm{~min}$, and 30 cycles of $95^{\circ} \mathrm{C}$ for $30 \mathrm{~s}, 56^{\circ} \mathrm{C}$ for $30 \mathrm{~s}$, and $72{ }^{\circ} \mathrm{C}$ for $30 \mathrm{~s}$ for IL-24 (381 bp) and GAPDH (264bp). The GAPDH RT-PCR products were used as controls of sample loading for normalisation between samples.

Enzyme-linked immunosorbent assay. The expression of IL-24 in cell-culture media and tumour xenografts was measured with a commercial human IL-24 ELISA kit following the manufacturer's instructions (Biomedical Laboratories, Piscataway, NJ, USA). Cells were treated with Ki67-ZD55-IL-24, ZD55-IL-24, Ki67-ZD55, and ZD55-EGFP, respectively. The conditioned media were harvested on day 3 and stored at $-70{ }^{\circ} \mathrm{C}$ until the assay. The homogenates were centrifuged at 12000 r.p.m., and the supernatants were collected. Diluted standards and supernatants containing IL-24 were added at $100 \mu \mathrm{l}$ per well in 96-well plates coated with anti-IL-24 monoclonal antibody. The optical density of the plates was finally measured at a wavelength of $450 \mathrm{~nm}$.

Immunocytochemical staining. Cells were fixed with $4 \%$ paraformaldehyde onto glass coverslips. After washing with phosphate-buffered saline (PBS), the cells were incubated with anti-Ki67 antibody for $24 \mathrm{~h}$, and then incubated with horseradish peroxidase-conjugated secondary antibody for $1 \mathrm{~h}$ followed by colorimetric detection using 3,3'-diaminobenzidine (DAB).

Cytopathic effect assay. Cells were plated in a 96- or a 48 -well plate and treated with ZD55-EGFP, Ki67-ZD55, ZD55-IL-24, or Ki67-ZD55-IL-24, respectively. At 72 or $120 \mathrm{~h}$ after treatment, the cells were washed, fixed with paraformaldehyde, and stained with crystal violet (Amresco, Solon, OH, USA).

Cell viability assay. Cells were plated in 96-well plates and transiently transfected with various adenoviruses. At the indicated times, the medium was removed and fresh medium containing 3-(4,5-dimethylthiazol-2-yl)-2,5-diphenyltetrazolium bromide (MTT, $0.5 \mathrm{mg} \mathrm{ml}^{-1}$ ) was added to each well. The cells were incubated at $37^{\circ} \mathrm{C}$ for $4 \mathrm{~h}$, and then an equal volume of solubilisation solution $(0.01 \mathrm{~N} \mathrm{HCl}$ in $10 \%$ SDS $)$ was added to each well and mixed thoroughly. The absorbance from the plates was read on an ELX-800 spectrometer (Bio-Tek Instruments Inc., Winooski, VT, USA) at $570 \mathrm{~nm}$.

Cell migration and invasion assays. Cell migration and invasion assays were performed using a modified two-chamber migration apparatus with a pore size of $8 \mu \mathrm{m}$ (Corning, Lowell, MA, USA). For the migration assays, the underside of a transwell filter was coated with $10 \mu \mathrm{g} \mathrm{ml}^{-1}$ fibronectin from human plasma (Sigma-Aldrich, St Louis, MO, USA) at $37^{\circ} \mathrm{C}$ overnight. In brief, after transfection for $6 \mathrm{~h}$, the adenovirus-transfected melanoma cells $\left(5 \times 10^{4}\right)$ were seeded into the upper chambers, and medium containing $10 \%$ fetal bovine serum filled the lower compartment. After incubation for $12 \mathrm{~h}$ at $37^{\circ} \mathrm{C}$ in a humidified atmosphere containing $5 \% \mathrm{CO}_{2}$, the cells in the upper chamber were mechanically removed with a cotton swab. The filters were fixed 
in methanol and stained with crystal violet. The cells that migrated into the lower surface were counted in five microscopic fields per filter using a $10 \times$ objective in at least three independent experiments. For the invasion assays, we performed a similar procedure using Matrigel-coated (Sigma-Aldrich) transwell chambers. The cells that suspended into the well bottom were quantified after incubation for $24 \mathrm{~h}$. The cells that migrated to the bottom surface of the membrane were subjected to microscopic inspection and then photographed randomly at $\times 100$ magnification.

Western blot analysis. The cells were harvested from the plates, and aliquots of cell extracts were separated on a $12 \%$ SDS-polyacrylamide gel. The proteins were then transferred onto a nitrocellulose membrane and incubated overnight at $4{ }^{\circ} \mathrm{C}$ with the following rabbit polyclonal antibodies: anti-IL-24 (Gen Hunter C-corporation, Nashville, TN, USA), anti-caspase-3, anti-Bcl-xl, anti-Bax, anti-Mcl-1 (Cell Signaling, Beverly, MA, USA), anti- $\beta$ actin, and anti-E1A antibodies (Santa Cruz Biotechnology, Santa Cruz, CA, USA), respectively. Membranes were then washed and incubated with alkaline phosphatase-conjugated secondary antibodies in tris-buffered saline containing Tween-20 for $2 \mathrm{~h}$ and developed using the NBT/BCIP color substrate (Promega). The densities of the bands on the membrane were scanned and analysed with ImageJ (LabWorks Software, UVP Upland, CA, USA).

Evaluation of apoptosis. Apoptosis was determined by staining the cells with annexin V-FITC and propidium iodide (PI) using the Annexin V-FITC Apoptosis Detection Kit (BD Pharmingen, San Diego, CA, USA). A375 cells were treated with Ki67-ZD55-IL-24, Ki67-ZD55, ZD55-IL-24, or ZD55-EGFP for $96 \mathrm{~h}$ and then harvested and washed twice with cold PBS. The prepared cells were resuspended in binding buffer $(10 \mathrm{~mm} \mathrm{HEPES} / \mathrm{NaOH}$ ( $\mathrm{pH} 7.4$ ), $140 \mathrm{mM} \mathrm{NaCl}$, and $2.5 \mathrm{mM} \mathrm{CaCl}_{2}$ ) at a concentration of $1 \times 10^{6}$ cells per $\mathrm{ml}$. Then, $5 \mathrm{ml}$ each of annexin V-FITC (Pharmingen) and PI were added to these cells, which were analysed by a FACStar flow cytometer (Becton Dickinson, Franklin Lakes, NJ, USA).

Cell-cycle analysis. For cell-cycle analysis, cells were seeded in 6 -well plates at a concentration of $2 \times 10^{5}$ cells per $4 \mathrm{ml}$ of medium per well. After 1 day, the cells were treated with Ki67-ZD55-IL-24, ZD55-IL-24, Ki67-ZD55, ZD55-EGFP, and PBS. At $72 \mathrm{~h}$, the cells were harvested, washed in PBS, and fixed in ice-cold 100\% ethanol for $12 \mathrm{~h}$ at $4{ }^{\circ} \mathrm{C}$. After washing, the cells were counted and the number of cells was adjusted. The cells were incubated in RNase $\left(0.4 \mathrm{mg} \mathrm{ml}^{-1}\right)$ for $1 \mathrm{~h}$, and then cellular DNA was stained with PI solution (final concentration: $4 \mu \mathrm{g} \mu \mathrm{l}^{-1}$ ) for $10 \mathrm{~min}$. The cell-cycle distribution of cell samples was determined by flow cytometric analysis (FACS SCAN; BD Biosciences, Heidelberg, Germany), and the data were analysed using FCS-express software (De novo software, Thornhill, ON, Canada). The percentages of cells at the G0/G1, S, and G2/M stages were calculated using the multicycle software.

Xenograft tumour model in nude mice. Male BALB/c nude mice at 4-5 weeks old were obtained from the Shanghai Experimental Animal Center of the Chinese Academy of Sciences (Shanghai, China) and quarantined for 1 week before tumour implantation. Animal welfare and experimental procedures were carried out strictly in accordance with the 'Guide for the Care and Use of Laboratory Animals' (National Research Council, 2011). The xenograft tumour model was established by subcutaneously injecting A375 cells $\left(2 \times 10^{6}\right)$ into the right flank of each mouse. When tumours reached $100-150 \mathrm{~mm}^{3}$, the mice were divided randomly into five groups ( 8 mice per group) and were treated by intratumoral injections of ZD55-EGFP, Ki67-ZD55, ZD55-IL-24, or Ki67-ZD55-IL-24 $\left(5 \times 10^{8}\right.$ PFU per dose per day $)$ for 3 consecutive days or with PBS as a control. The tumour was monitored every week by measuring the tumour size using calipers, direct observation of animal behaviour, and body weight monitoring for 30 days. The tumour volume was calculated by the following formula: $V\left(\mathrm{~mm}^{3}\right)=$ length $\times$ width $^{2} \times 1 / 2$. The animal weight was measured at day 0 and remeasured every 3 days until the end of the experiments. The body weights of mice treated with PBS, ZD55-EGFP, Ki67-ZD55, ZD55-IL-24, or Ki67-ZD55-IL-24 were expressed as a percentage of baseline. Data represent mean \pm standard error of the mean (s.e.m.) $(n=6)$. At the end of the experiment, tumours were harvested for additional analyses as described below. Differences in tumour growth and animal weight were tested for statistical significance.

Immunohistochemistry staining. Tumours were harvested and fixed in $10 \%$ formalin, embedded in paraffin, and cut into $4-\mathrm{mm}$ sections. Deparaffinized tumour sections were treated with $3 \%$ $\mathrm{H}_{2} \mathrm{O}_{2}$ for $10 \mathrm{~min}$ to block endogenous peroxidases and incubated with blocking serum (goat serum) at room temperature for $30 \mathrm{~min}$. Immunohistochemistry was carried out with an anti-IL-24 antibody. After incubation with an anti-mouse secondary antibody, the expression of IL-24 was detected with DAB (Sigma, St Louis, $\mathrm{MO}$, USA) by enhancement with an avidin-biotin reaction $\mathrm{ABC}$ kit (Vector Laboratories, Burlingame, CA, USA). Tissue sections stained without primary antibody served as negative controls. The slides were then counterstained with haematoxylin. For evaluation of IL-24-positive fractions, at least 200 cells were counted from 6 different regions and the mean number was determined.

Haematoxylin and eosin staining and the TUNEL assay. Tumours were harvested and fixed in $4 \%$ paraformaldehyde, embedded in paraffin, and cut into $4-\mu \mathrm{m}$ sections. For histopathological analysis, the paraffin sections of tumours were stained with haematoxylin and eosin ( $\mathrm{H} \& \mathrm{E})$. Apoptotic cells in tumour tissue sections were quantified using an in situ apoptosis detection kit (Roche, Indianapolis, IN, USA). Formalin-fixed paraffin-embedded sections were dewaxed before being permeabilised with proteinase $\mathrm{K}$ for $15 \mathrm{~min}$ at room temperature. Endogenous peroxidase was blocked with $3 \% \mathrm{H}_{2} \mathrm{O}_{2}$, and sections were incubated with equilibration buffer and terminal deoxynucleotidyl transferase enzyme. Finally, the sections were incubated with an antidigoxigenin-peroxidase conjugate. Peroxidase activity in each tissue section was visualised by the application of DAB. Using microscopy, 6 fields were randomly selected from every sample and 100 cells were randomly selected from every field. The apoptotic rate $=($ number of total apoptotic cells $/ 100) \times 100 \%$.

Statistical analysis. Data were expressed as the mean \pm standard deviation (s.d.). After comparison of the homogeneity of variance (homogeneity of variance test), one-way analysis of variance was used to determine whether there were any significant differences among the means of the different treatment groups, followed by the Student-Newman-Kuels (SNK) multiple comparison test to determine the difference between two groups within the multiple groups demonstrating significant differences. All statistical analyses were performed by using the statistical software (SPSS Base 13.0 for Windows, SPSS Inc., Chicago, IL, USA). Probability values $(P)<0.05$ were considered as significant.

\section{RESULTS}

Ki67-ZD55 mediates efficient delivery of IL-24 in A375 melanoma cells. We first examined the efficiency of Ki67-ZD55mediated delivery of IL-24 in A375 and M14 cells treated with ZD55-EGFP, Ki67-ZD55, ZD55-IL-24, or Ki67-ZD55-IL-24. The PBS mock-treated cells were used as controls. Interleukin-24 expression levels were determined by RT-PCR, western blotting, and enzyme-linked immunosorbent assay (ELISA) analyses. After treatment for $48 \mathrm{~h}$, IL-24 was stably overexpressed in A375 and 
M14 cells and no discernible difference of IL-24 expression was observed in cells treated with either Ki67-ZD55-IL-24 or ZD55-IL-24 (Figure 1A and B), demonstrating that Ki67-ZD55 can mediate a high and stable expression of IL-24. Furthermore, we detected similar expression of the adenoviral E1A protein in Ki67-ZD55-IL-24- and ZD55-IL-24-infected cells (Figure 1B), indicating that Ki67-ZD55-IL-24 replicates productively in melanoma cells.

In line with the RT-PCR and western blotting results, after Ki67-ZD55-IL-24 or ZD55-IL-24 infection, A375 cells produced a significantly greater concentration of IL-24 $\left(498 \pm 8.7 \mathrm{pg} \mathrm{ml}^{-1}\right.$ or $305 \pm 7.2 \mathrm{pg} \mathrm{ml}^{-1}$, respectively) than Ki67-ZD55 $\left(90 \pm 1.7 \mathrm{pg} \mathrm{ml}^{-1}\right)$ or ZD55-EGFP (86 $\left.\pm 2.1 \mathrm{pg} \mathrm{ml}^{-1}\right)(P<0.05$, Figure $1 \mathrm{C})$.

Ki67 expression in a melanoma cell line and a normal cell line. To assess Ki67 expression in a melanoma cell line, melanoma A375 and M14 cells and normal lung fibroblast NHLF cells were frozen and subjected to immunohistochemical analysis of Ki67. As shown in Figure 2A, human melanoma cell lines A375 and M14 showed highly positive Ki67 expression, while Ki67 expression in NHLF cells was very low.

Potent cytopathic effect induced by the combination of Ki67-ZD55-IL-24. Cells were treated with ZD55-EGFP, Ki67ZD55, ZD55-IL-24, or Ki67-ZD55-IL-24, respectively, and were stained with crystal violet 7 days later. As shown in Figure 2B, the Ki67-ZD55-IL-24 treatment induced a potent cytopathic effect in A375 and M14 cells in a dose-dependent manner. For each concentration gradient tested, treatment with Ki67-ZD55-IL-24 produced a greater cytopathic effect in both cell lines than that of ZD55-IL-24 or Ki67-ZD55 treatment alone. A significant suppression of cell proliferation was not observed in normal NHLF cells.

Ki67-ZD55-IL-24 results in enhanced loss of cell viability. To further evaluate the kinetics of cytotoxicity induced by Ki67-ZD55-IL-24, A375 and M14 cells were respectively treated with ZD55-EGFP alone, Ki67-ZD55 alone, ZD55-IL-24 alone, or Ki67-ZD55-IL-24 alone at a multiplicity of infection (MOI) of 10 for different periods of time. As shown in Figure 2C, the cytopathic effect of Ki67ZD55-IL-24 on melanoma cells was induced in a time-dependent manner with the most severe growth inhibition seen after 4 days of treatment. As controls, single treatment with either Ki67-ZD55 or
ZD55-IL-24 induced a much weaker cytopathic effect in melanoma cells than in those treated with Ki67-ZD55-IL-24 $(P<0.05)$.

To investigate whether Ki67-ZD55-IL-24 inhibits the proliferation of melanoma cells, A375 and M14 cells were respectively plated in 96-well plates and treated with different dosages of ZD55EGFP alone $(0.1,1,10$, or 50 MOI), Ki67-ZD55 alone $(0.1,1,10$, or 50 MOI), ZD55-IL-24 alone (0.1, 1, 10, or 50 MOI), or Ki67-ZD55IL-24 $(0.1,1,10$, or $50 \mathrm{MOI})$. Four days following treatment, cell viability was determined by the MTT assay. As shown in Figure 2, Ki67-ZD55-IL-24 treatment led to enhanced inhibition of A375 cell proliferation in a dose-dependent manner. In addition, for each concentration tested, treatment with Ki67-ZD55-IL-24 resulted in greater inhibition than that of Ki67-ZD55 or ZD55-IL-24 treatment alone $(P<0.05)$.

To evaluate the safety of Ki67-ZD55-IL-24, a normal cell line, NHLF, was treated with ZD55-EGFP, Ki67-ZD55, ZD55-IL-24, or Ki67-ZD55-IL-24, respectively. After 4 days, drug-induced cytotoxicity was determined by the MTT assay. As shown in Figure 2C, Ki67-ZD55-IL-24 treatment had similar toxic effects compared with ZD55-IL-24 or Ki67-ZD55 treatment, but did not have any overlapping toxicity in normal cells.

Ki67-ZD55-IL-24 decreases cells migration and invasion in human melanoma cells. We further investigated cell motility by migration and invasion assays. In the cell migration assay, our data revealed that melanoma cells cultured in supernatant from Ki67ZD55-IL-24 treatment migrated slower than melanoma cells cultured in supernatant from ZD55-IL-24 or Ki67-ZD55 treatment $(P<0.05)$ (Figure 3A). In the cell invasion assay, Ki67-ZD55-IL-24 inhibited the invasive ability of melanoma cells in a Matrigelcoated Boyden chamber compared with the control cells (Figure 3B). These findings illustrated that Ki67-ZD55-IL-24 could decrease the migration and invasion of melanoma cells in vitro.

Ki67-ZD55-IL-24 increases apoptosis in melanoma cells via a mitochondria-mediated apoptosis pathway. To elucidate the mechanism underlying apoptosis observed in the Ki67-ZD55-IL-24 treatment, we monitored the protein levels of Bcl-xl, Mcl-1, Bax, and caspase- 3 by western blot analysis after treatment of A375 cells with ZD55-EGFP, Ki67-ZD55, ZD55-IL-24, or Ki67-ZD55-IL-24. We observed that Bcl-xl and Mcl-1 levels were decreased with
A

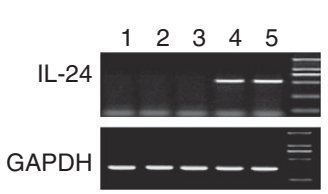

A375

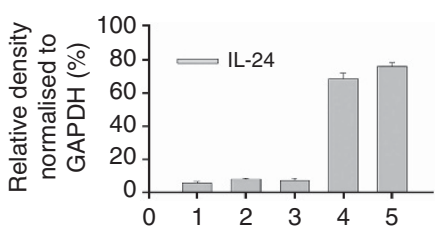

M14
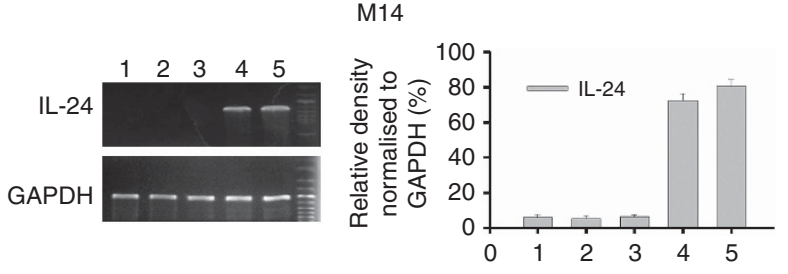

B

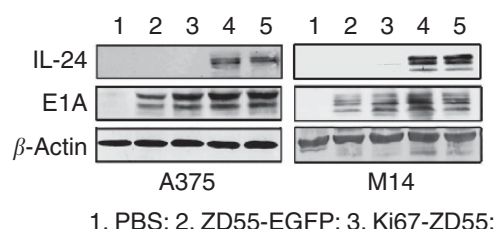

4. ZD55-IL24; 5. Ki67-ZD55-IL24

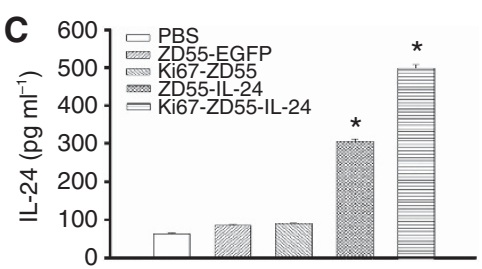

1. PBS; 2. ZD55-EGFP; 3. Ki67-ZD55;4. ZD55-IL24; 5. Ki67-ZD55-IL24

Figure 1. Ki67-ZD55-IL-24 induced the efficient delivery of IL-24 in melanoma cells. A375 and M14 cells were treated with ZD55-EGFP, Ki67-ZD55, ZD55-IL-24, or Ki67-ZD55-IL-24, respectively. (A) After 48 h, IL-24 mRNA levels were analysed by RT-PCR. Cycle numbers were optimised in several experiments to determine the linear phase of the PCR. The corresponding GAPDH levels are shown as internal controls. Quantitation of changes in band densities was performed by ImageJ analysis software. (B) IL-24 and E1A protein levels were analysed by western blotting using anti-IL-24 and anti-E1A antibodies. $\beta$-Actin was used as a loading control. (C) The cell-culture medium was harvested 3 days after infection and measured with a commercial IL-24 ELISA kit. Data are shown as mean \pm s.d. ${ }^{\star} P<0.05$. 
A
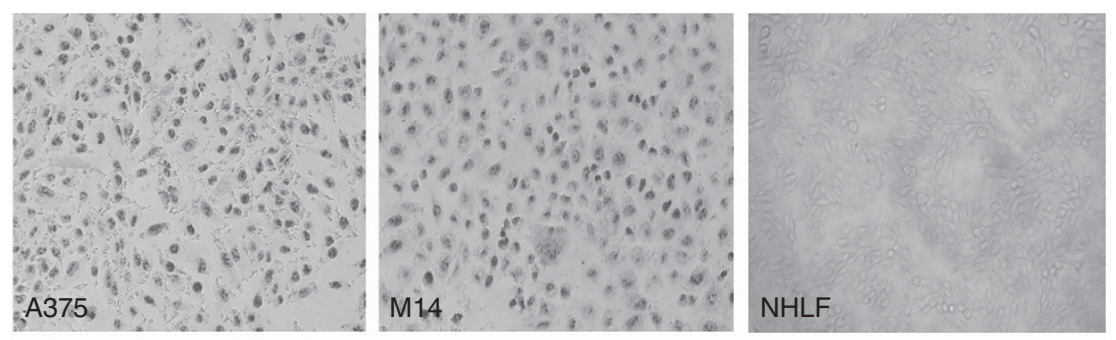

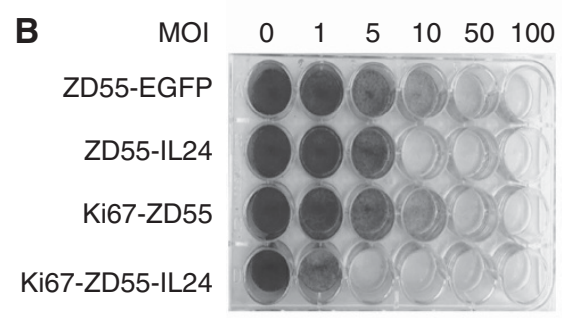

A375

C
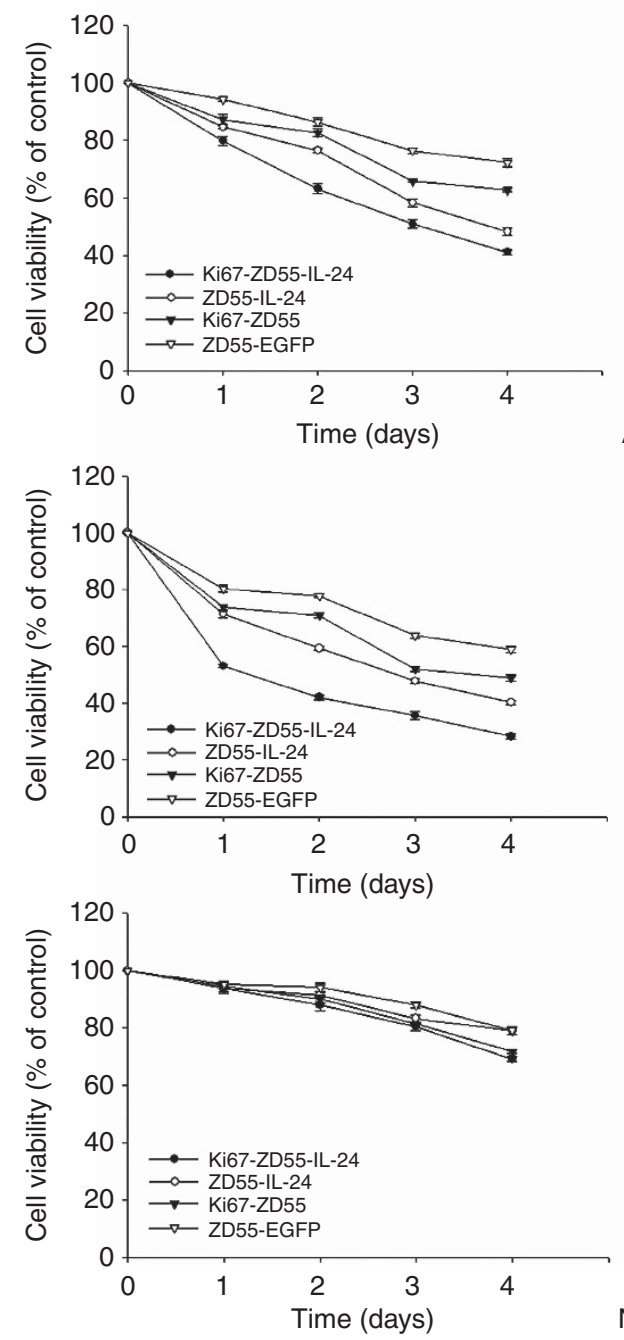

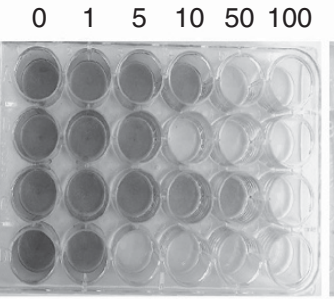

M14

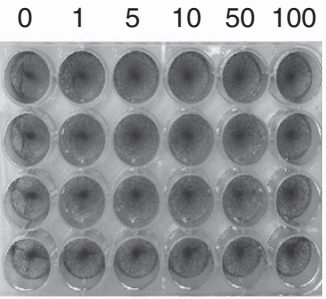

NHLF
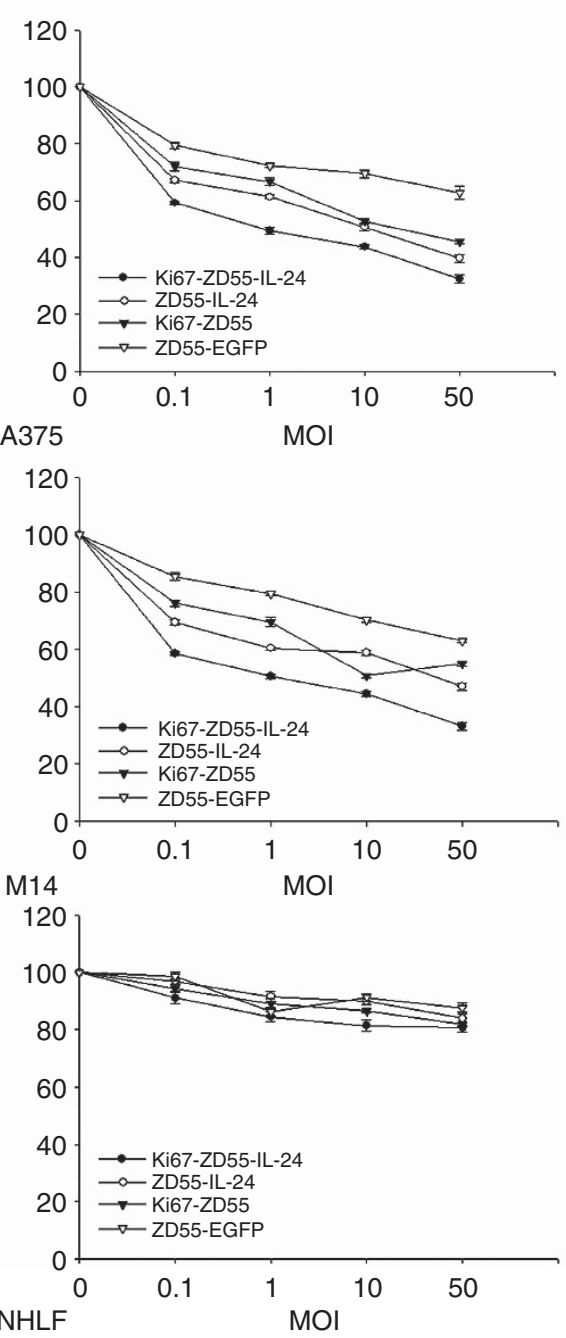

Figure 2. Ki67-ZD55-IL-24 inhibited the growth of melanoma cells but did not have overlapping toxicity in normal cells. (A) The expression of Ki67 in melanoma cells and normal NHLF cells was assessed by immunocytochemical analysis. Representative pictures for different cell groups (original magnification, $\times 400$ ). (B) Cytolytic activities of Ki67-ZD55-IL-24 in A375, M14, and NHLF cells. Cells were plated at a density of $10^{5}$ cells per 6-cm dish and treated with ZD55-EGFP, Ki67-ZD55, ZD55-IL-24, or Ki67-ZD55-IL-24, respectively, at the indicated dosage. They were stained with crystal violet at day 7. The presence of staining indicates the presence of live cells. (C) Melanoma A375 and M14 cells and normal NHLF cells were respectively infected with ZD55-EGFP, Ki67-ZD55, ZD55-IL-24, or Ki67-ZD55-IL-24 at an MOI of 10. On days 1, 2, 3, and 4 post infection, the cells were subjected to the MTT assay. A375 and M14 cells and normal NHLF cells were treated with ZD55-EGFP, Ki67-ZD55, ZD55-IL-24, or Ki67-ZD55-IL-24, respectively, at the indicated dosages. The cells were subjected to the MTT assay on the fourth day after treatment. The results are expressed as mean \pm s.d. (error bars) as the percentage of untreated control cells $(n=6)$. 
A
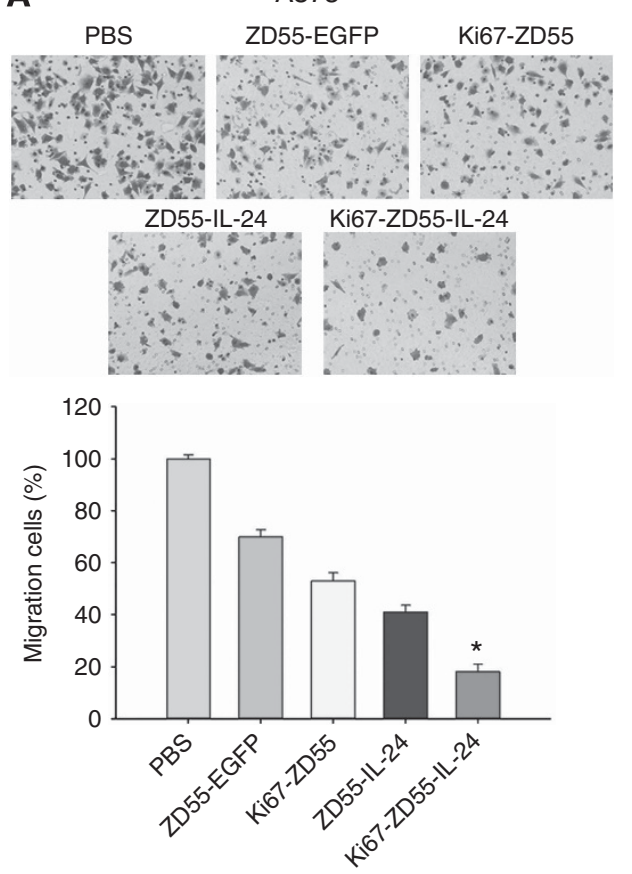

B

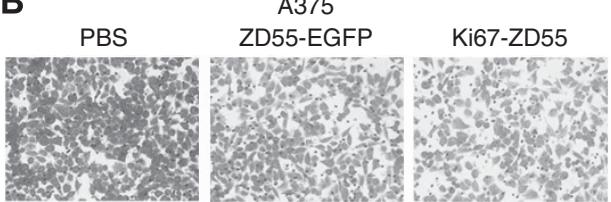

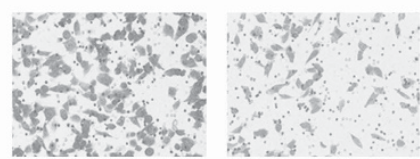

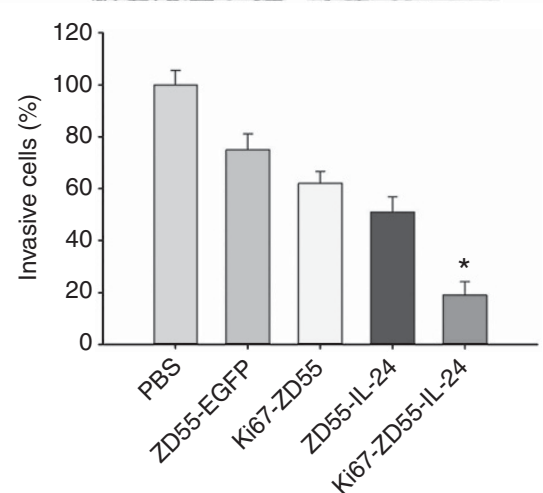

M14
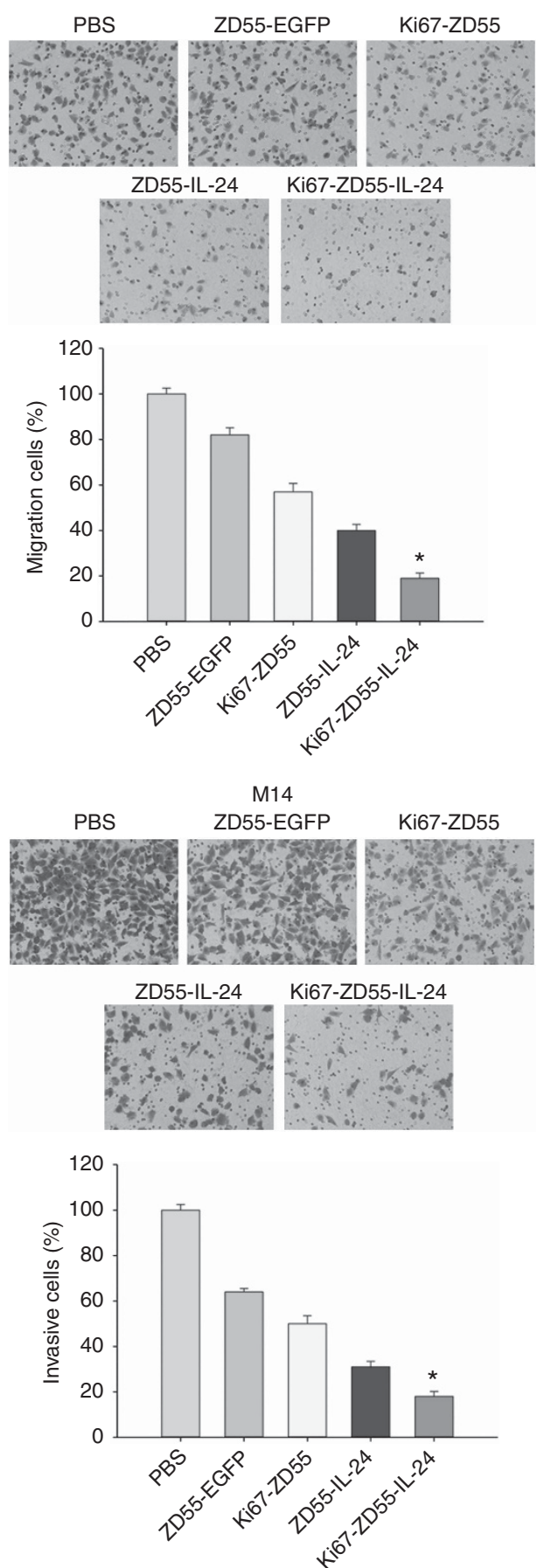

Figure 3. Ki67-ZD55-IL-24 regulates melanoma cell migration and invasion. (A) The cell migration assay was executed after Ki67-ZD55-IL-24 treatment in melanoma cells. Ki67-ZD55-IL-24 decreased the ability of the cells to migrate through the Boyden chamber compared with the control cells. Data are shown as mean \pm s.d. ${ }^{*} P<0.05$. (B) The cell invasion assay was executed after Ki67-ZD55-IL-24 treatment in melanoma cells. Ki67-ZD55-IL-24 decreased the ability of invasion through the Boyden chamber compared with the control cells. Data are shown as mean \pm s.d. $\star P<0.05$.

ZD55-IL-24 or Ki67-ZD55 treatment, but was further reduced with Ki67-ZD55-IL-24 treatment (Figure 4). In addition, PBS, ZD55EGFP, or Ki67-ZD55 alone did not significantly increase Bax expression, whereas ZD55-IL-24 or Ki67-ZD55-IL-24 significantly increased Bax expression. Accordingly, cleaved caspase- 3 was significantly increased in the Ki67-ZD55-IL-24 treatment group (Figure 4). These results suggested that Ki67-ZD55-IL-24 enhanced apoptosis in melanoma cells by a mitochondria-mediated apoptosis pathway.
Ki67-ZD55-IL-24 contributes to apoptosis and cell-cycle arrest in melanoma cells. To test whether this enhanced cytotoxicity caused by Ki67-ZD55-IL-24 was due to increased apoptosis, we examined the apoptotic changes in A375 cells after different treatment schemes. A375 cells were treated with ZD55-EGFP, Ki67-ZD55, ZD55-IL-24, or Ki67-ZD55-IL-24 at an MOI of 10 for $96 \mathrm{~h}$. Enhanced permeability of the cellular membrane, an early sign of apoptosis, was assessed by flow cytometric analysis using annexin V-FITC staining. After treatment for $96 \mathrm{~h}$, PBS treatment 
alone only caused membrane instability in a small number of cells with ratios slightly above the basal level in A375 cells, whereas ZD55-EGFP, Ki67-ZD55, and ZD55-IL-24 treatments caused 19.5, 25.5, and $34.0 \%$ of A375 cells to lose membrane integrity. The Ki67-ZD55-IL-24 treatment resulted in 51.2\% of A375 cells to lose membrane integrity, which was significant $(P<0.05)$ (Figure $5 \mathrm{~A}$ ).

The cell-cycle phase was assayed by flow cytometry after PI staining of the fixed cells. The level of A375 cells at the G2/M phase was greater in the Ki67-ZD55-IL-24 group (43.10\%) than in the ZD55-IL-24 (16.36\%), Ki67-ZD55 (6.31\%), and ZD55-EGFP $(3.18 \%)$ groups (Figure 5B). Similar results were also observed in M14 cells (data not shown).

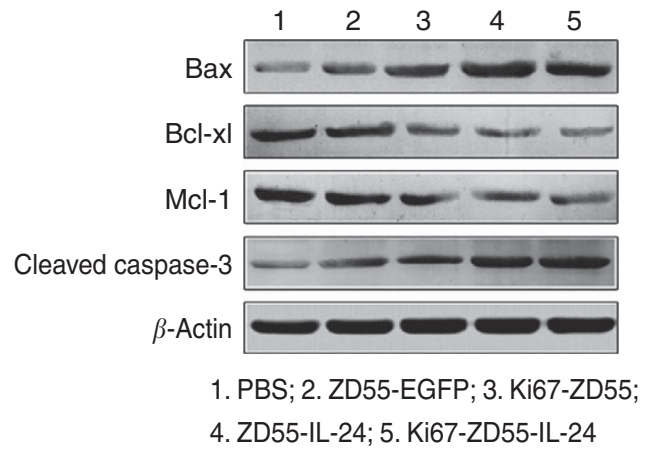

Figure 4. Ki67-ZD55-IL-24 increases apoptosis in melanoma cells via a mitochondria-mediated apoptosis pathway. Melanoma cells were treated with ZD55-EGFP, Ki67-ZD55, ZD55-IL-24, or Ki67-ZD55-IL-24, and PBS treatment was used as a negative control. After treatment for $72 \mathrm{~h}$, the cell extracts were subjected to western blot analysis for activation of caspase-3. The expression levels of the anti-apoptotic proteins $\mathrm{Bcl}-\mathrm{xl}$ and $\mathrm{Mcl}-1$ and the pro-apoptotic protein Bax were also examined. $\beta$-actin was used as a loading control $(n=6)$.
Anti-tumour efficacy of Ki67-ZD55-IL-24 in nude mice. Based on the in vitro data above, we investigated the effects of Ki67ZD55-IL-24 treatment on tumour growth in vivo. Nude mice received $2 \times 10^{6}$ A375 cells subcutaneously. When the tumour reached $100-150 \mathrm{~mm}^{3}$, mice were divided randomly into five groups (8 mice per group) and received ZD55-EGFP, Ki67-ZD55, ZD55-IL-24, or Ki67-ZD55-IL-24 $\left(5 \times 10^{8}\right.$ PFU per dose for 3 consecutive days) $(n=6)$. A group of mice receiving A375 cells and then a PBS injection was included as the control. Nude mice were killed at 30 days after tumour cell inoculation. The tumours were removed for additional analyses as described below. In the control group receiving PBS, tumours displayed rapid and continued outgrowth during the course of the experiment. The mean tumour size was $5718 \pm 2058.87 \mathrm{~mm}^{3}$. The mean tumour size in the ZD55EGFP treatment group was $3531.3 \pm 913.20 \mathrm{~mm}^{3}$, while it was $2438.5 \pm 622.71 \mathrm{~mm}^{3}$ in the Ki67-ZD55 treatment group, and $1648.72 \pm 478.54 \mathrm{~mm}^{3}$ in the ZD55-IL-24 treatment group. The mean tumour size of the Ki67-ZD55-IL-24 treatment group was $1287.8 \pm 290.12 \mathrm{~mm}^{3}$, which was much smaller than that of the ZD55-IL-24 treatment group $(P=0.037)$ and the Ki67-ZD55 treatment group $(P=0.026)$ (Figure 6A). The toxicities were assessed by direct observation of animal behaviour and body weight monitoring. The mice in both the ZD55-IL-24 and Ki67ZD55-IL-24 groups tolerated the regimens well. The treatments did not seem to have obvious adverse impacts on their activity level or body weight (Figure 6B). In addition, the Ki67-ZD55-IL-24-treated tumours displayed the least net tumour weight at the end of this experiment $(P<0.05)$ (Figure $6 \mathrm{C}$ and $\mathrm{D})$.

To verify the therapeutic effect of Ki67-ZD55-IL-24 in vivo, we also analysed the expression of IL-24 and E1A by immunohistochemistry and western blotting. As shown in Figure 6E, IL-24 was stably overexpressed in A375 melanoma xenografts in nude mice, and no discernible difference of IL-24 expression was observed in cells treated with either Ki67-ZD55-IL-24 alone or ZD55-IL-24 alone, demonstrating that Ki67-ZD55 or ZD55 can

A
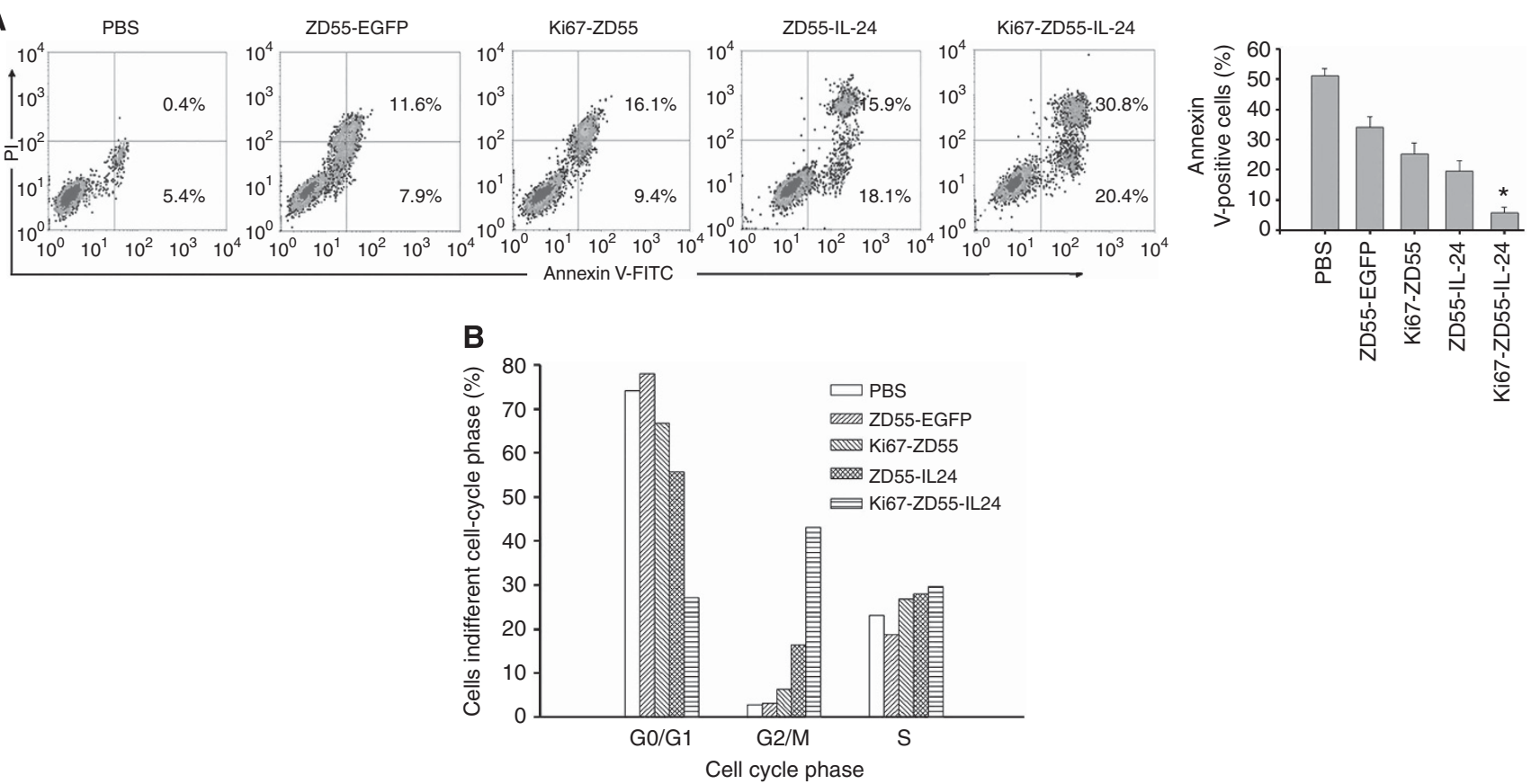

Figure 5. Ki67-ZD55-IL-24 contributes to apoptosis and cell-cycle arrest in melanoma cells. A375 cells were treated with ZD55-EGFP, Ki67-ZD55, ZD55-IL-24, or Ki67-ZD55-IL-24, and PBS treatment was used as a negative control. (A) After $96 \mathrm{~h}$, the cells were stained with FITC-labelled annexin $\mathrm{V}$ and immediately analysed by flow cytometry. Data are presented as mean \pm s.d. of the percentage of annexin-positive cells from three independent experiments. The asterisk (*) designates $P<0.05$ vs the ZD55-IL-24-, Ki67-ZD55-, or ZD55-EGFP-treated groups ( $n=6$ ). (B) Cell-cycle distribution of cell samples was determined by flow cytometric analysis, and data were analysed using the FCS-express software. The percentage of cells at the G0/G1, S, and G2/M stages was calculated using the multicycle software. 

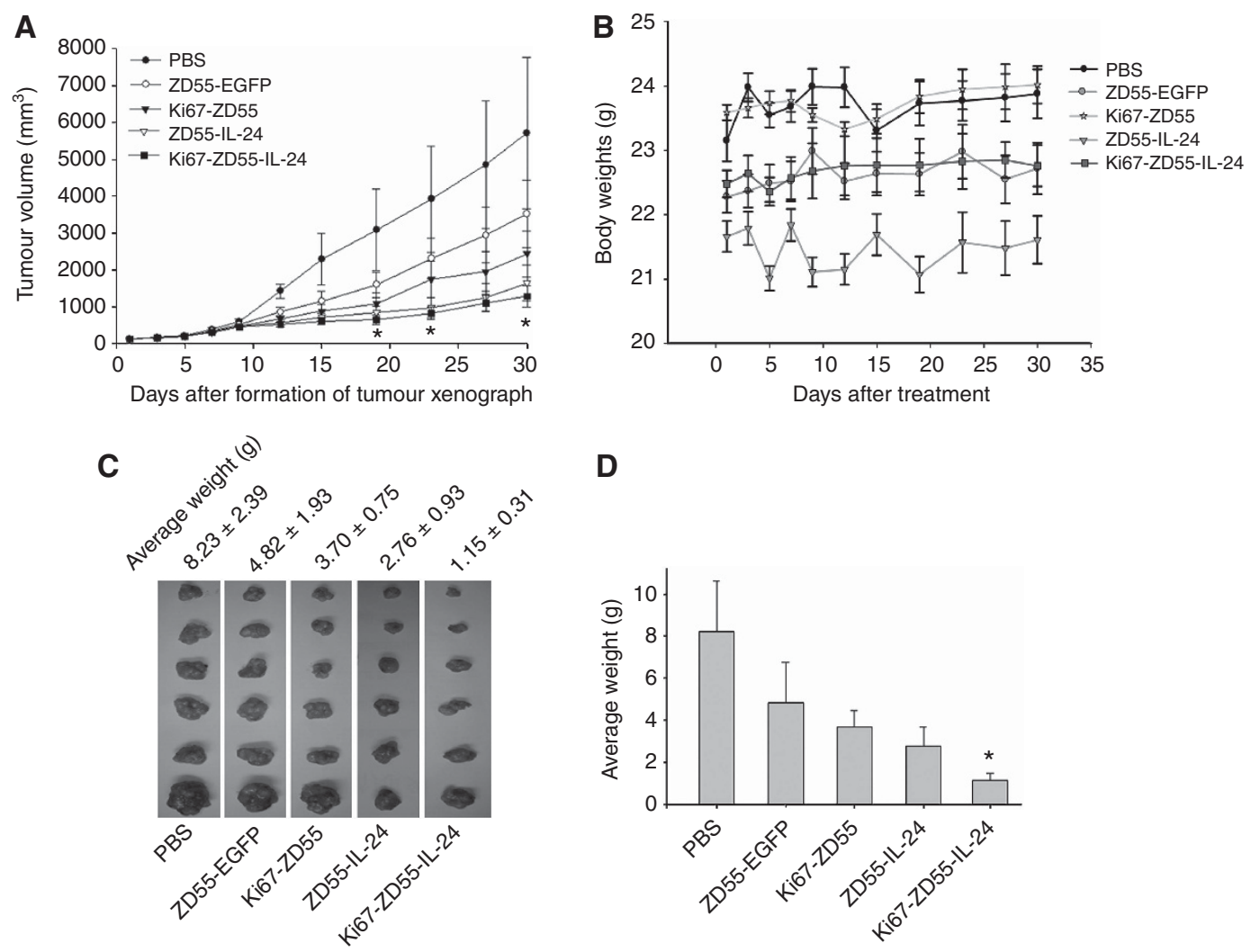

D
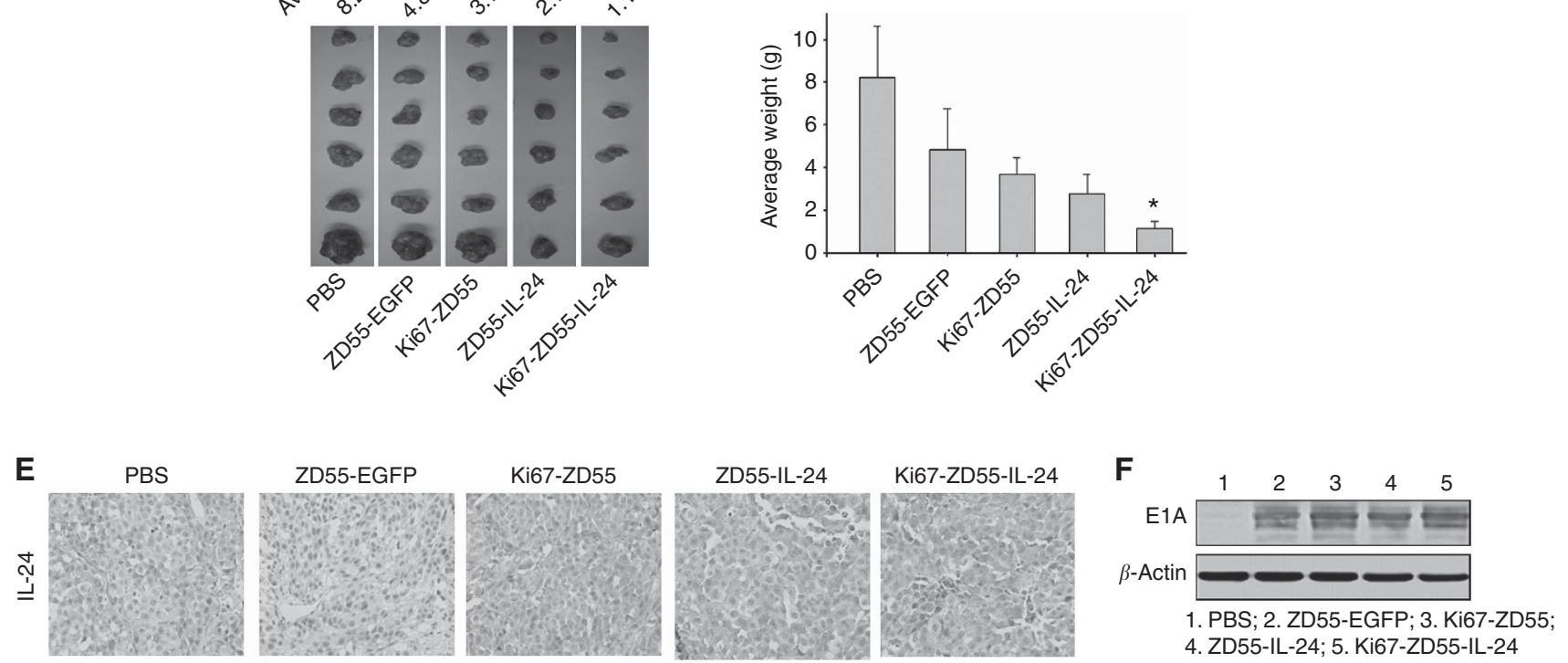

Figure 6. Anti-tumour activity of Ki67-ZD55-IL-24 in the A375 xenograft model. Tumours were established by injecting A375 cells subcutaneously into the right flank of nude mice. When tumours reached $100-150 \mathrm{~mm}^{3}$, mice were divided randomly into five groups (8 mice per group) and were treated by intratumoral injections of ZD55-EGFP, Ki67-ZD55, ZD55-IL-24, or Ki67-ZD55-IL-24 (5 × $10^{8}$ PFU per dose per day) with three consecutive daily injections or with PBS as a control. (A) The tumour size was measured, and the tumour volume was calculated. Error bars represent the s.d. Data are expressed as means of tumour volume \pm s.d. (error bars). The asterisk (*) indicates $P<0.05$ vs the ZD55-IL-24-, Ki67-ZD55-, or ZD55-EGFP-treated groups $(n=6)$. (B) The body weights of mice treated with PBS, ZD55-EGFP, Ki67-ZD55, ZD55-IL-24, or Ki67-ZD55-IL-24 were expressed as a percentage of baseline. Tumour-bearing mice were administered intravenously with PBS, ZD55-EGFP, Ki67-ZD55, ZD55-IL-24, or Ki67-ZD55-IL-24, respectively, every 3 days until the end of the experiments. Data represent mean \pm s.e.m. ( $n=6$ ).

(C) At the end of this experiment, tumours from the mice were removed and weighed. (D) Data are expressed as means of tumour volume \pm s.d. (error bars). The asterisk (*) indicates $P<0.05$ vs the ZD55-IL-24-, Ki67-ZD55-, or ZD55-EGFP-treated groups ( $n=6$ ). (E) The expression of IL-24 in the A375 xenograft model was assessed by immunohistochemical analysis. Ki67-ZD55-IL-24 induced the number of IL-24-positive cells (original magnification, $\times 400)(n=6)$. (F) Western blotting detected E1A expression in the lysed tumour cells.

mediate a high and stable expression of IL-24. Moreover, E1A expression was detected in the Ki67-ZD55-IL-24, ZD55-IL-24, Ki67-ZD55, and ZD55-EGFP treatment groups but not in the PBS treatment group, which indicated that Ki67-ZD55-IL-24 could replicate in tumour cells (Figure 6F).

Ki67-ZD55-IL-24 induces apoptosis in melanoma xenografts in nude mice. We further studied the pathological changes by $\mathrm{H} \& \mathrm{E}$ staining in tumours injected with Ki67-ZD55-IL-24. Mice were killed after observation, and their tumours were collected and examined pathologically by $\mathrm{H} \& \mathrm{E}$ staining (Figure 7A). Many large necrotic regions were found in the tumours obtained from the treated groups, especially in the Ki67-ZD55-IL-24-treated group. But in the PBS group, the cancer cells grew controllably with only small focal areas of necrosis. The apoptotic cells in the tumour sections were analysed by TUNEL staining. TUNEL staining showed markedly increased numbers of apoptotic cells in the Ki67-ZD55-IL-24 group, which was significant compared with the other three treatment groups (Figure 7B).

\section{DISCUSSION}

Melanoma is an extremely aggressive and fatal tumour. It is notoriously resistant to all of the current therapeutic modalities, including chemotherapy and radiotherapy. Suppressed apoptosis and extraordinary invasiveness are the distinctive features that contribute to the malignancy of melanoma (Eberle et al, 2007). Thus, there is an urgent need to develop effective new therapeutic strategies that decrease tumour recurrence and distant metastasis.

We have previously demonstrated that ZD55-IL-24 in combination with chemotherapy exhibits a strong tumour suppression 

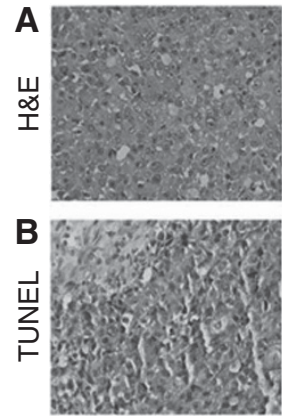

PBS
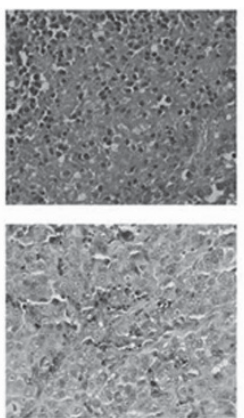

ZD55-EGFP
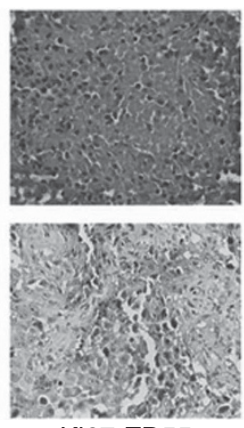

Ki67-ZD55
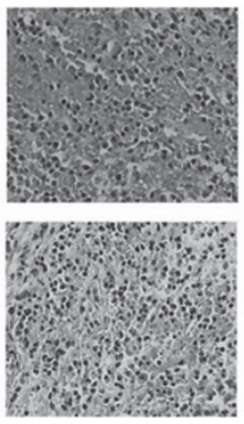

ZD55-IL-24

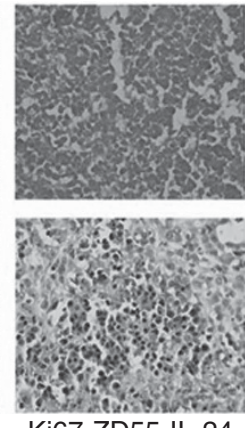

Ki67-ZD55-IL-24

Figure 7. Induction of apoptosis after treatment with Ki67-ZD55-IL-24 in the A375 xenograft model. Tumour sections were excised and analysed by H\&E staining and TUNEL staining. (A) Tumour sections were processed for H\&E staining. Scale bar $=100 \mu \mathrm{m}$. (B) Representative photomicrographs showing TUNEL staining in the implanted tumour (original magnification, $\times 400)(n=6)$.

effect for melanoma cells. To optimise the efficacy of the CRAd ZD55, the identification of tumour-selective promoter elements that can differentiate between normal and cancer cells is of immense importance. One strategy to achieve the desired tumour selectivity of CRAds is the use of tumour-specific transcriptional response elements (promoters) to control the expression of essential early viral genes for replication, such as E1A (Liu and $\mathrm{Gu}, 2006$ ).

We recently cloned a powerful cancer-specific promoter, the Ki67 promoter, which has been granted patent application approval in China (patent number: ZL200810022295.7). The Ki67 promoter is a TATA-less, GC-rich type of promoter (Wang et al, 2011). The promoter of the Ki67 gene as an excellent tumourselective promoter with desirable specificity and efficiency that may further restrict transgene expression to tumour cells and improve the targeting of gene therapy (Keam et al, 2011) (Figure 8). Compared with the hTERT and survivin promoters, the Ki67 core promoter possesses greater transcription activity and more desirable tumour selectivity (Pei et al, 2012). In addition, our laboratory has demonstrated that Ki67-ZD55-IL-24 could improve the anti-tumour effects of the alkylating agent temozolomide in melanoma cells (Jiang et al, 2013).

In this study, we utilised the Ki67 promoter to replace the native promoter of E1A on the basis of the CRAd ZD55 and generated a double-regulated CRAd carrying the therapeutic gene IL-24, Ki67ZD55-IL-24. We examined the efficacy and toxicity of Ki67-ZD55IL-24-mediated gene therapy in melanoma cells. Western blot analysis in vitro and in vivo confirmed the greater expression of E1A protein in cells treated with ZD55-EGFP, Ki67-ZD55, ZD55IL-24, or Ki67-ZD55-IL-24. We observed elevated expression of transgenic IL-24 only in Ki67-ZD55-IL-24- or ZD55-IL-24-treated melanoma cells. These results indicated that Ki67-ZD55-IL-24 or ZD55-IL-24 could efficiently replicate in melanoma cells. Consistent with the ability to replicate in tumour cells, the MTT assay showed that Ki67-ZD55-IL-24 could specifically induce cytopathic effects in melanoma cells. CRAds represent a novel class of therapeutic agents in cancer treatment. CRAds enter cells through infection and can kill both proliferating and quiescent tumour cells (Eriksson et al, 2007; Yano et al, 2013). The tumour growth inhibition curve also indicated that Ki67-ZD55-IL-24 treatment could effectively inhibit xenograft growth in nude mice. The Ki67ZD55-IL-24-treated tumours displayed the least net tumour weight at the end of the experiment.

The fate of tumour cells is largely dependent on the expression of pro-apoptotic (e.g., Bax) vs anti-apoptotic genes (e.g., Bcl-xl) (Eberle et al, 2008). To further elucidate the mechanism of Ki67-ZD55-IL-24-induced cell death, we determined the effect of Ki67-ZD55-IL-24 on the levels of Bcl-xl, Bax, Mcl-1, and caspase-3 proteins by western blot analysis in melanoma cells. We found that

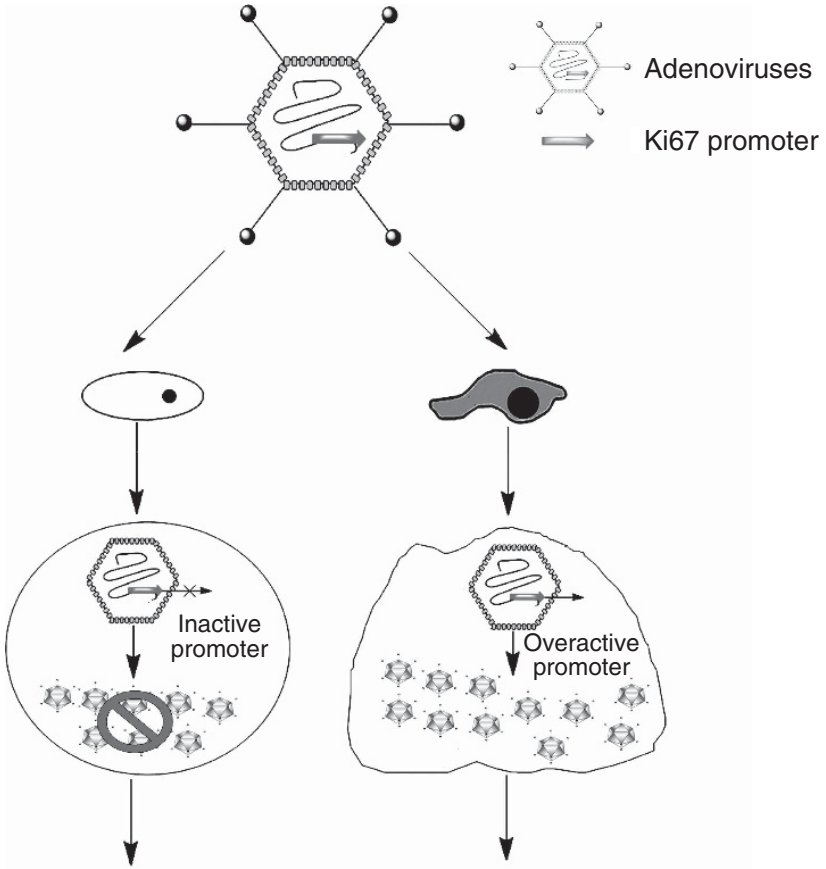

No viral replication

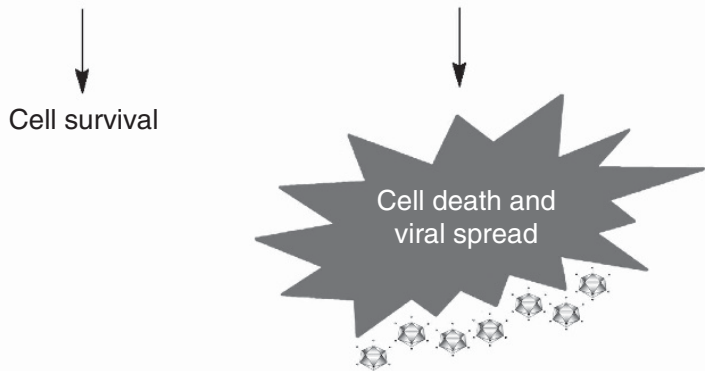

Figure 8. Use of the Ki67 promoter to direct lytic viral replication to tumour cells. The adenoviral E1A gene was placed under the control of a promoter, the Ki67 promoter (dashed arrow), which is preferentially overactive in a given tumour cell type. This modification drives viral replication in tumour cells but not in normal cells.

Ki67-ZD55-IL-24 dramatically decreased Bcl-xl and Mcl-1 levels, significantly increased Bax, and induced a much stronger activation of caspase-3. These results suggested that Ki67-ZD55IL-24 could induce changes in the levels and ratio of pro-apoptotic to anti-apoptotic proteins in melanoma cells. These changes may contribute to Ki67-ZD55-IL-24-induced apoptosis. 
In this study, we showed that the Ki67 promoter could regulate E1A expression and viral replication, confer tumour-specific replication of a CRAd, and enable transcriptional targeting of melanoma cells. The novel CRAd Ki67-ZD55-IL-24 exerted a powerful anti-tumour effect both in vitro and in melanoma tumour xenografts on nude mice. One major limitation of the use of the $\mathrm{Ki} 67$ promoter to regulate adenovirus replication is that it is not always active in melanoma. It is unlikely that all of the melanoma cells will express the specific tumour marker Ki67 because melanoma is heterogeneous; thus, the remaining non-expressing normal cells may have a selective advantage.

\section{ACKNOWLEDGEMENTS}

This project is supported by grants from the National Natural Science Foundation of China (No. 81372916, 81372460, and 81101702), the Science and Technology Project of Xuzhou city (No. XM13B084), the 'Six Talent Peaks' Project of Jiangsu Province (No. 2013-WSN-014), the Key University Science Research Project of Jiangsu Province (No. 11KJA320002 and 12KJA320001), the Science and Technology Department of Jiangsu province (No. BK2013348 and BK2011207), and Xuzhou Medical Young Talents Project.

\section{CONFLICT OF INTEREST}

The authors declare no conflict of interest.

\section{REFERENCES}

Beresford MJ, Wilson GD, Makris A (2006) Measuring proliferation in breast cancer: practicalities and applications. Breast Cancer Res 8: 216.

Bischoff JR, Kirn DH, Williams A, Heise C, Horn S, Muna M, Ng L, Nye JA, Sampson-Johannes A, Fattaey A, McCormick F (1996) An adenovirus mutant that replicates selectively in p53-deficient human tumour cells. Science 274: 373-376.

Chai L, Liu S, Mao Q, Wang D, Li X, Zheng X, Xia H (2012) A novel conditionally replicating adenoviral vector with dual expression of IL-24 and arresten inserted in E1 and the region between E4 and fiber for improved melanoma therapy. Cancer Gene Ther 19: 247-254.

Cheang MC, Chia SK, Voduc D, Gao D, Leung S, Snider J, Watson M, Davies S, Bernard PS, Parker JS, Perou CM, Ellis MJ, Nielsen TO (2009) Ki67 index, HER2 status, and prognosis of patients with luminal B breast cancer. J Natl Cancer Inst 101: 736-750.

Cody JJ, Douglas JT (2009) Armed replicating adenoviruses for cancer virotherapy. Cancer Gene Ther 16: 473-488.

Eager R, Harle L, Nemunaitis J (2008) Ad-MDA-7; INGN 241: a review of preclinical and clinical experience. Expert Opin Biol Ther 8: 1633-1643.

Eberle J, Fecker LF, Hossini AM, Kurbanov BM, Fechner H (2008) Apoptosis pathways and oncolytic adenoviral vectors: promising targets and tools to overcome therapy resistance of malignant melanoma. Exp Dermatol 17: 1-11.

Eberle J, Kurbanov BM, Hossini AM, Trefzer U, Fecker LF (2007) Overcoming apoptosis deficiency of melanoma-hope for new therapeutic approaches. Drug Resist Updat 10: 218-234.

Emdad L, Lebedeva IV, Su ZZ, Gupta P, Sauane M, Dash R, Grant S, Dent P, Curiel DT, Sarkar D, Fisher PB (2009) Historical perspective and recent insights into our understanding of the molecular and biochemical basis of the antitumour properties of mda-7/IL-24. Cancer Biol Ther 8: 391-400.

Eriksson M, Guse K, Bauerschmitz G, Virkkunen P, Tarkkanen M, Tanner M, Hakkarainen T, Kanerva A, Desmond RA, Pesonen S, Hemminki A (2007)
Oncolytic adenoviruses kill breast cancer initiating CD44 + CD24-/low cells. Mol Ther 15: 2088-2093.

Jiang G, Jiang AJ, Cheng Q, Tian H, Li LT, Zheng JN (2013) A dual-regulated oncolytic adenovirus expressing interleukin- 24 sensitizes melanoma cells to temozolomide via the induction of apoptosis. Tumour Biol 34: 1263-1271.

Jiang H, Su ZZ, Lin JJ, Goldstein NI, Young CS, Fisher PB (1996) The melanoma differentiation associated gene mda-7 suppresses cancer cell growth. Proc Natl Acad Sci USA 93: 9160-9165.

Keam B, Im SA, Lee KH, Han SW, Oh DY, Kim JH, Lee SH, Han W, Kim DW, Kim TY, Park IA, Noh DY, Heo DS, Bang YJ (2011) Ki-67 can be used for further classification of triple negative breast cancer into two subtypes with different response and prognosis. Breast Cancer Res 13: R22.

Liu E, Telem DA, Warner RR, Dikman A, Divino CM (2011) The role of Ki-67 in predicting biological behavior of goblet cell carcinoid tumour in appendix. Am J Surg 202: 400-403.

Liu XY (2006) Targeting gene-virotherapy of cancer and its prosperity. Cell Res 16: 879-886.

Liu XY, Gu JF (2006) Targeting gene-virotherapy of cancer. Cell Res 16: 740. National Research Council (2011) Guide for the Care and Use of Laboratory Animals. National Academy Press: Washington, DC, USA.

Pei DS, Qian GW, Tian H, Mou J, Li W, Zheng JN (2012) Analysis of human Ki-67 gene promoter and identification of the Sp1 binding sites for Ki-67 transcription. Tumour Biol 33: 257-266.

Rioux-Leclercq N, Turlin B, Bansard J, Patard J, Manunta A, Moulinoux JP, Guille F, Ramee MP, Lobel B (2000) Value of immunohistochemical Ki-67 and $\mathrm{p} 53$ determinations as predictive factors of outcome in renal cell carcinoma. Urology 55: 501-505.

Sinnberg T, Lasithiotakis K, Niessner H, Schittek B, Flaherty KT, Kulms D, Maczey E, Campos M, Gogel J, Garbe C, Meier F (2009) Inhibition of PI3K-AKT-mTOR signaling sensitizes melanoma cells to cisplatin and temozolomide. J Invest Dermatol 129: 1500-1515.

Vaisanen A, Kuvaja P, Kallioinen M, Turpeenniemi-Hujanen T (2011) A prognostic index in skin melanoma through the combination of matrix metalloproteinase-2, Ki67, and p53. Hum Pathol 42: $1103-1111$

Wang MJ, Pei DS, Qian GW, Yin XX, Cheng Q, Li LT, Li HZ, Zheng JN (2011) p53 regulates Ki-67 promoter activity through p53- and Sp1-dependent manner in HeLa cells. Tumour Biol 32: 905-912.

Yano S, Tazawa H, Hashimoto Y, Shirakawa Y, Kuroda S, Nishizaki M, Kishimoto H, Uno F, Nagasaka T, Urata Y, Kagawa S, Hoffman RM, Fujiwara T (2013) A genetically engineered oncolytic adenovirus decoys and lethally traps quiescent cancer stem-like cells in S/G2/M phases. Clin Cancer Res 19: 6495-6505.

Yerushalmi R, Woods R, Ravdin PM, Hayes MM, Gelmon KA (2010) Ki67 in breast cancer: prognostic and predictive potential. Lancet Oncol 11: 174-183.

Yu DC, Chen Y, Dilley J, Li Y, Embry M, Zhang H, Nguyen N, Amin P, Oh J, Henderson DR (2001) Antitumour synergy of CV787, a prostate cancer-specific adenovirus, and paclitaxel and docetaxel. Cancer Res 61: 517-525.

Zhao L, Gu J, Dong A, Zhang Y, Zhong L, He L, Wang Y, Zhang J, Zhang Z, Huiwang J, Qian Q, Qian C, Liu X (2005) Potent antitumour activity of oncolytic adenovirus expressing mda-7/IL-24 for colorectal cancer. Hum Gene Ther 16: 845-858.

Zheng JN, Ma TX, Cao JY, Sun XQ, Chen JC, Li W, Wen RM, Sun YF, Pei DS (2006) Knockdown of Ki-67 by small interfering RNA leads to inhibition of proliferation and induction of apoptosis in human renal carcinoma cells. Life Sci 78: 724-729.

This work is published under the standard license to publish agreement. After 12 months the work will become freely available and the license terms will switch to a Creative Commons AttributionNonCommercial-Share Alike 3.0 Unported License. 